\title{
XQuery Containment in Presence of Variable Binding Dependencies
}

\author{
Li Chen \\ San Diego Supercomputer Center \\ 9500 Gilman Drive, La Jolla, CA 92093 \\ lichen@sdsc.edu
}

\author{
Elke A. Rundensteiner \\ CS Department, Worcester Polytechnic Institute \\ Worcester, MA 01609 \\ rundenst@cs.wpi.edu
}

\begin{abstract}
Semantic caching is an important technology for improving the response time of future user queries specified over remote servers. This paper deals with the fundamental query containment problem in an XQuery-based semantic caching system. To our best knowledge, the impact of subtle differences in XQuery semantics caused by different ways of specifying variables on query containment has not yet been studied. We introduce the concept of variable binding dependencies for representing the hierarchical element dependencies preserved by an XQuery. We analyze the problem of XQuery containment in the presence of such dependencies. We propose a containment mapping technique for nested XQuery in presence of variable binding dependencies. The implication of the nested block structure on XQuery containment is also considered. We mention the performance gains achieved by a semantic caching system we build based on the proposed technique.
\end{abstract}

\section{Categories and Subject Descriptors}

F.3 [Theory of Computation]: Logics and Meanings of Programs; I.1.1 [Computing Methodologies]: Expressions and Their Representation; H.2.8 [Information Systems]: Database Applications

\section{General Terms}

XQuery containment mapping algorithm and theory

\section{Keywords}

XQuery Containment, variable binding dependency

\section{INTRODUCTION}

Due to its fundamental role in many database applications such as query optimization and information integration [16], the problem of query containment has received considerable attention over the past few decades. With the initial focus on relational queries, researchers have recently begun to study the containment problem for various fragments of XPath [13, 1, 22, 12] and XQuery [9, 18, 10]. The key technique of containment mapping for relational queries [4] has been extended in the new contexts to derive mappings between navigation pattern trees and nested XQuery Copyright is held by the International World Wide Web Conference Committee (IW3C2). Distribution of these papers is limited to classroom use, and personal use by others.

WWW 2005, May 10-14, 2005, Chiba, Japan.

ACM 1-59593-046-9/05/0005. constructs. It has been commonly recognized that extended containment mapping is central for minimizing XML queries $[23,9]$, and for reformulating queries in a mediator system [9] or a peer-to-peer environment [18].

\subsection{Motivation}

This work is motivated by the promising application of semantic caching for answering XML queries using cached XML views $[6,8]$. The idea of semantic caching is that the (mobile) client maintains both the semantic descriptions and associated answers of previous queries in its cache, in the hope of being able to reuse them to speed up the processing of subsequent queries.

An XQuery-based semantic caching system named ACEXQ has been proposed [6, 7] for facilitating XQuery processing in the Web environment. The main techniques exploited by ACE-XQ include the containment mapping approach for nested XQuery, XQuery rewriting, and a multi-granularity replacement strategy. With [8] focussed on the proposed replacement strategy and the cache performance evaluation, we introduce, in this paper, the fundamental query containment technique underlining ACE-XQ which is the first comprehensive practical semantic cache solution for handling nested conjunctive XQuery.

\subsection{The Related Work}

In the XML setting, extensive research has focussed on the query containment problem for regular path expressions on general cyclic graph databases [3], tree pattern queries and XPath queries over XML data $[13,1,22,12]$. Especially the containment problem for XPath and tree pattern queries has attracted a lot of attention recently due to the fundamental role they play in many XML query languages.

Different fragments of XPath have been targeted by different works. A well recognized core XPath fragment includes child axis '/', descendant axis "//", branching "[ ]", and wildcard '*'. It is shown in [13] that query containment for this fragment, denoted $X P^{\{*, / /,[]\}}$, is coNP-complete. If any of the three constructs "//", "[ ]", and '*' is dropped, query containment is PTIME. The essence of their containment mapping technique is the polynomial-time tree homomorphism algorithm ${ }^{1}$, which serves as a sufficient but not necessary condition for containment of $X P^{\{*, / /,[]\}}$ in general. On the other hand, if tag variables and equality testing are allowed, query containment is NP-complete. The complexity increases to $\Pi_{2}^{p}$ with disjunctions added. We refer

\footnotetext{
${ }^{1}$ Tree homomorphism and tree embedding are exchangeable.
} 
the readers to $[1,22,12]$ for discussions of the containment complexity results under different XPath fragments.

However, research on the containment problem for XQuery is still in its infancy. Besides using XPath expressions as the navigation mechanism, XQuery also employs other query constructs such as FLWR expressions and the nesting of query blocks. These features make XQuery more expressive than XPath. On the other hand, they also impose new difficulties on the containment problem. Specifically, difficulties arise since an XQuery cannot simply be represented by a navigation tree pattern. Hence containment mapping based on tree homomorphism alone is no longer sufficient for determining XQuery containment.

To our best knowledge, the containment of nested XQuery has so far been studied only in [9], [18], and [10]. [9] exploits XQuery containment for query optimization. It utilizes containment mapping for identifying redundant navigation patterns in a query and later for collapsing them to minimize the query. In [18], the containment of nested XQuery is researched for the purpose of rewriting queries posted on one peer to be answered by another peer. [10] studies the complexity of the problem regarding completeness.

Targeting different goals, these three works exploit different approaches. The containment mapping technique proposed in [9] essentially extends tree homomorphism between navigation patterns with additional requirements for mapping the equality-based where-conditions, groupby_id and groupby_value variables. In [18], two types of mappings, i.e., a query-head embedding $E_{\text {head }}\left(Q_{1}, Q_{2}\right)$ and a query-body embedding $E_{\text {body }}\left(Q_{2}, Q_{1}\right)$, are employed as the sufficient conditions for deriving $Q_{1} \sqsubseteq Q_{2}$ (assuming $Q_{1}$ and $Q_{2}$ are two nested XQueries). $E_{\text {head }}$ embeds the block structure of $Q_{1}$ into that of $Q_{2}$ while $E_{b o d y}$ embeds the navigation pattern of $Q_{2}$ into that of $Q_{1}$. In [10], containment of nested XQuery is defined based on XML instance containment. The theoretical complexity result for methods that ensure completeness is established.

Among these three works, [10] presents an approach that guarantees completeness (i.e., no false negative answers). In answering-queries-using-views scenarios, it is commonly considered more crucial to guarantee the soundness while the completeness is often ignored to avoid the high complexity. For example, the containment of nested XQuery in general is coNEXPTIME when ensuring completeness [10].

In contrast, [9] and [18] attempt to provide more practical containment mapping techniques by extending tree homomorphism with additional mapping conditions. In [9], a technique is proposed for identifying redundant navigation within one query. It considers the mapping of equality-based where-conditions and that of variables distinguished by the set or bag semantics they each represent. However, all return expressions are considered as black-box functions and ignored in the containment mapping process. This containment mapping technique is hence a not suitable foundation for determining the containment relationship between two queries. This is obvious considering the fact that whether the direct bindings of variable $v$ or subelements obtained from further navigation of $v$ 's bindings are returned does make a major difference in the query result.

Furthermore, neither of the two techniques considers the effect of dependencies among variable bindings on the query result and consequently on the containment result. In Section 1.3, we give examples of subtle differences in XQuery semantics caused by different dependencies among the specified variables. Since these two techniques have failed to address the critical effect of such differences on the query containment result, we propose our containment mapping approach which provides sufficient mapping conditions for correctly deriving the containment decision.

\subsection{Problem Analysis}

In this work, we target the containment problem for nested XQuery. We consider a core fragment of XQuery that allows nested blocks, conjunctive equality-based conditions, set and bag semantics. Disjunctions, negations, universal quantifier and tag variables are not considered. This XQuery fragment is the same as that being studied in [9]. [18] and [10] study a subset of this fragment as they exclude the bag semantics.

\begin{tabular}{|c|c|}
\hline 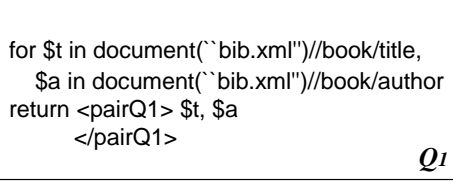 & $\begin{array}{l}\text { for } \$ b \text { in document }(. .) / / \text { book, } \\
\$ \text { t in } \$ \mathrm{~b} / \text { title, } \$ \mathrm{a} \text { in } \$ \mathrm{~b} / \text { author } \\
\text { where some } \$ \mathrm{p} \text { in } \$ \mathrm{~b} / \text { price } \\
\text { satisfies } \$ \mathrm{p}=30 \\
\begin{array}{l}\text { return }<\text { pairQ2> } \$ \mathrm{t}, \$ \mathrm{a} / \text { last } \\
\quad<\text { pairQ2> }\end{array}\end{array}$ \\
\hline $\begin{array}{l}\text { for } \$ \mathrm{~b} \text { in document(..)//book } \\
\text { return }<\text { pairQ3> } \\
\{\text { for } \$ \mathrm{t} \text { in } \$ \mathrm{~b} / \text { title, } \$ \text { a in } \$ \mathrm{~b} / \text { author } \\
\text { return } \$ \mathrm{t}, \$ \mathrm{a}\} \\
\quad</ \text { pairQ3 }>\end{array}$ & 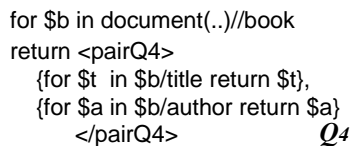 \\
\hline
\end{tabular}

Figure 1: Example Queries

Now let us consider the example queries in Figure 1. All four queries $Q_{i}(i=1 . .4)$ specify $\$ t$ and $\$ a$ and return their bindings in the results. Suppose the input document bib.xml is shown at the left top corner in Figure 2, we can see that their results $R_{Q i}(i=1 . .4)$ (also shown in Figure 2) are all different due to the subtle differences in their variable specifications and nested block structures. Suppose that the DTD for bib.xml specifies <!Element book(title, author*, publisher?, price?)>. $R_{Q 1}$ contains six title and author pairs derived from all combinations of the $\$$ t and $\$$ a bindings documentwide regardless of whether the paired title and author elements belong to the same book. In contrast, the $\$$ t and $\$ a$ bindings in $Q_{2}$ are specified based on $\$ \mathrm{~b}$. Therefore, the title and author elements corresponding to different book parents do not appear in the same pair in $R_{Q 2}$. For example, $t 2$ is paired with $a 1$ and $a 2$ but not with $a 3$ in $R_{Q 2}$.

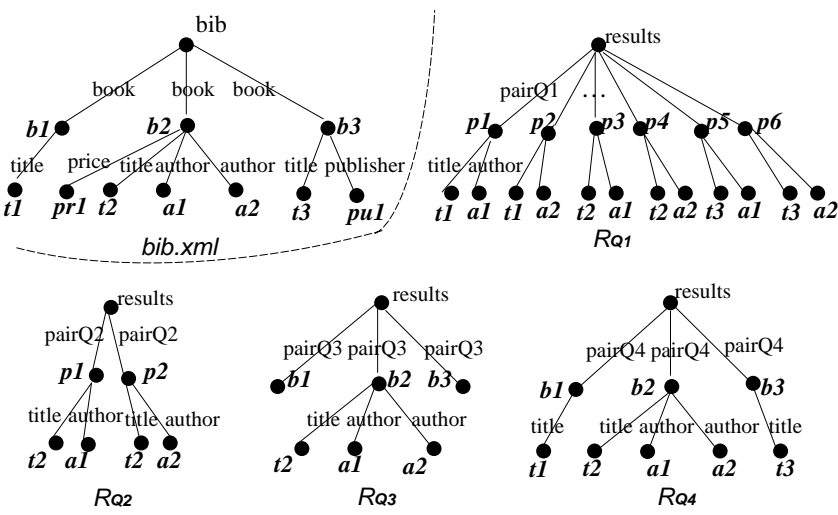

Figure 2: bib.xml and Example Query Results

The differences in the structure of $R_{Q 1}$ versus that of $R_{Q 2}$ can be intuitively explained by the differences in specifying 
variable dependencies in $Q_{1}$ and $Q_{2}$. That is, the variables $\$ t$ in $Q_{2}$ (denoted by $\$ t_{Q 2}$ ) and $\$ a_{Q 2}$ are defined based on $\$ b_{Q 2}$, while $\$ t_{Q 1}$ and $\$ a_{Q 1}$ are based on $\$ r$ (i.e., the default root variable bound to the root element of document("bib.xml")).

We first explain the effect of variable dependencies on the resulting query result for $Q_{2}$. When constructing the result $R_{Q 2}$, since $\$ t_{Q 2}$ and $\$ a_{Q 2}$ are defined in the same query block, the corresponding new element $\langle$ pair $Q 2\rangle$ is produced for each tuple in the cartesian product of the bindings of $\$ t_{Q 2}$ and $\$ a_{Q 2}$. Due to the way how $\$ t_{Q 2}$ and $\$ a_{Q 2}$ are specified, the bindings of $\$ t_{Q 2}$ and $\$ a_{Q 2}$ derived from the same binding of $\$ b_{Q 2}$ preserve the sibling $\langle$ title $\rangle\langle$ author $\rangle$ element associations under the same parent book element. Such hierarchical data dependencies in the source XML are preserved in the intermediate variable bindings based on which the query result is constructed. In this case, each pairQ 2 element in $R_{Q 2}$ combines bindings of $\$ t_{Q 2}$ and bindings of $\$ a_{Q 2}$ only if they share the same parent binding of $\$ b_{Q 2}$. In contrast, the sibling $\langle$ title $\rangle-\langle$ author $\rangle$ associations are not kept in the bindings of $\$ t_{Q 1}$ and $\$ a_{Q 1}$. $Q_{1}$ hence produces $\langle$ pair $Q 1\rangle$ elements based on the cartesian product of all the bindings of $\$ t$ and $\$ a$ regardless of their respective parent book elements. $Q_{2}$ hence preserves a finer hierarchy of element dependencies among its intermediate variable bindings than $Q_{1}$ does.

We now analyze the effect of such preserved dependencies on the containment result. Suppose that the containment mapping technique proposed in [18] is applied to $Q_{1}$ and $Q_{2}$ in Figure 2. $Q_{2} \sqsubseteq Q_{1}$ would be derived since both $E_{\text {head }}\left(Q_{2}, Q_{1}\right)$ and $E_{\text {body }}\left(Q_{1}, Q_{2}\right)$ can be established as illustrated in Figure $3^{2}$. To derive $Q_{2} \sqsubseteq Q_{1}$, this approach utilizes not only the navigation pattern based mapping represented by $E_{\text {body }}$, but also $E_{\text {head }}$ for checking if the variables returned by $Q_{2}$ are a subset of those returned by $Q_{1}$. However, whether such dependencies among variable bindings influence the query containment result has not been studied in either [18] or [9].

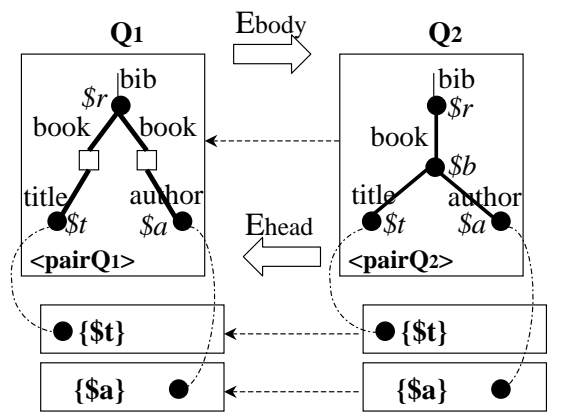

Figure 3: Illustration of Containment Mapping via $E_{\text {head }}$ and $E_{\text {body }}$

Assume $Q_{2}$ is answered using $R_{Q 1}$ based on the containment result $Q_{2} \sqsubseteq Q_{1}$. Then there is no way to re-group the returned bindings of $\$ t$ and $\$ a$ in $R_{Q 1}$ by their respec-

\footnotetext{
${ }^{2}$ As explained more in detail in [18], $E_{\text {head }}\left(Q_{2}, Q_{1}\right)$ embeds the nested block structure of $Q_{2}$ into that of $Q_{2}$. The dashed arrows denote the mappings between blocks within which the corresponding returned variables match. $E_{\text {body }}\left(Q_{1}, Q_{2}\right)$ embeds the navigation patterns (denoted by the bold tree edges) specified in $Q_{1}$ into those in $Q_{2}$. $E_{\text {body }}\left(Q_{2}, Q_{1}\right)$ but not $E_{\text {head }}\left(Q_{1}, Q_{2}\right)$ can be established. Hence $Q_{1} \nsubseteq Q_{2}$.
}

tive book parent elements as required by $Q_{2}$. Ignoring this requirement, the produced result of $Q_{2}$ would contain superfluous pairs, namely, $t 1-a 1, t 1-a 2, t 3-a 1$ and $t 3-a 2$.

\subsection{Our Contributions}

First, we address the problem of producing superfluous answers based on the query containment result when ignoring the effect of variable binding dependencies in the containment mapping process. Correspondingly we identify some important concepts and their connections, as illustrated in Figure 4, to this problem.

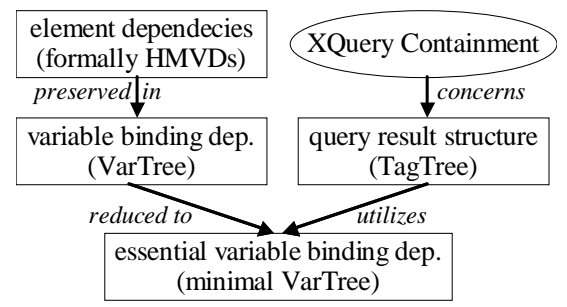

Figure 4: Connection between Preserving of Element Dependencies and XQuery Containment

The left hand side flow illustrates the preservation of data dependencies in the source XML in the intermediate bindings via the specification of variables. The right hand side flow represents the fact that XQuery containment needs to take the query result structure constructed based on the binding dependencies into consideration. Terms enclosed in the parentheses in Figure 4 will be introduced in Section 2.

Also, we realize that not all the intermediate binding dependencies preserved by a query are necessarily utilized in constructing the final result. Thus we call a subset of variable binding dependencies being utilized the essential ones via which both flows are connected.

Second, based on our problem analysis, we propose a containment mapping technique that considers the containment of the utilized binding dependencies in the query result. For this, we first decompose the input query and represent the two parts of its semantics, i.e., variable binding and result construction, by respective tree structures. Then we identify the binding dependencies that are preserved by the former and utilized by the latter. We call it variable minimization. Next we propose to employ three types of containment mappings for deriving the containment decision.

In sum, we will show that our containment mapping approach is more comprehensive than the prior works $[9,18$, $10]$ in that it deals with the effect of variable binding dependencies on the query containment result. In other words, it avoids deriving the query containment decision which may lead to producing superfluous answers for the contained query by using the result of the containing query. Like [9] and [18], our approach does not necessarily ensure completeness.

\subsection{Paper Outline}

The rest of the paper is organized as follows. In Section 2, we define the problem of XQuery containment in the presence of variable binding dependencies. Section 3 gives the overview of our approach. We describe the pre-step of query decomposition and minimization in Section 4. This is followed by our containment mapping technique in Section 5 . We show the query performance gains achieved by apply- 
ing the proposed technique in a semantic caching system in Section 6 and conclude in Section 7.

\section{PROBLEM DEFINITION}

In this section, we first introduce the notion of hierarchical multivalued dependencies (HMVDs) which represent a typical type of data dependencies in the source XML. Also, we define variable binding dependencies as the HMVDs being preserved by the query in the intermediate variable bindings. We then define the problem of nested XQuery containment in the presence of variable binding dependencies.

\subsection{Hierarchical Multivalued Dependencies}

It has been recently recognized that studying the extension of the traditional integrity constraints in the XML setting is both theoretically and practically meaningful. Several classes of integrity constraints including key constraints, path constraints, functional constraints, and inclusion constraints have been defined for XML [11]. The more advanced constraints such as the multivalued dependencies (aka tuple generating dependencies) have also been studied in $[19,2]$ with the goal to develop a normalization theory for XML and in [15] for mapping XML DTDs to relational schemas.

XPath containment in the presence of DTD constraints such as sibling constraints and functional constraints has been investigated in [11]. The semantics of an XPath query can be captured by a unary pattern tree in which only one node has its bindings returned as the result while others are matched but not returned. However in XQuery, even a single for clause may specify multiple variables which correspond to an n-ary $(n \geq 1)$ pattern tree. This is where the challenges arise for XQuery containment.

Let us first analyze the semantics of a single-block XQuery for the sake of simplicity. In a single-block XQuery that utilizes a FLWR expression, the return clause is invoked for all the cartesian product combinations of the variable bindings produced by the for clause. These combinations are determined based on how variables are defined based on others. As far as we know, no research has studied the implication of such dependencies on XQuery containment. This is the task of our work.

DeFinition 2.1. Given a DTD, suppose $\varepsilon$ is a set of binary edge relations between element type $e$ and its children element types, each labeled with the corresponding cardinality relationship 1, ?, * or $+{ }^{3}$. For any two descendant element types $x$ and $y$ of $e$, if either $x$ or $y$ has a multiple cardinality relationship (i.e., * or + ) with e, then we call the dependency among their corresponding elements in a conforming XML a hierarchical multivalued dependency (HMVD), denoted $e \rightarrow x \mid y$.

Recall that the notion of multivalued dependency (MVD) in relational databases defines that if a relation has two or more multivalued independent attributes (e.g., $x$ and $y$ ), every value of one attribute (e.g., $x$ ) must be repeated with every value of the other attribute (e.g., $y$ ). HMVD extends MVD in the sense that $e, x$ and $y$ are not attributes in a relation but element types in a DTD. If $e, x$ and $y$ are mapped to a 3-column relation and their bindings are unnested, then in each partition with an $e$ binding, every $x$ binding needs to be repeated with every $y$ binding.

\footnotetext{
${ }^{3} 1, ?, *$ and + respectively represent the $1-1,1-(0,1), 1-(0, m)$
} and $1-(1, m)(m \geq 1)$ mappings.

\subsection{Variable Binding Dependency}

For an XML document $D$, the dependencies among its elements which have multiple cardinality relationships with their respective parents can be represented by HMVDs. A query imposed against $D$ specifies a subset of HMVDs (direct or derived) to be preserved by its variable bindings.

Definition 2.2. Suppose a given query defines variable $v_{j}$ based on $v_{i}$, e.g., for $v_{j}$ in $v_{i}(/ / / /) p_{j}$, where $p_{j}$ is the relative XPath expression used for deriving $v_{j}$ 's bindings from each binding of $v_{i}$. We call this dependency of $v_{j}$ 's bindings on their respective $v_{i}$ bindings a variable binding dependency, denoted $v_{i} \stackrel{p_{j}}{\triangleright} v_{j}$.

For example, $\$ b \stackrel{\text { /title }}{\triangleright} \$ t$ and $\$ b \stackrel{\text { /author }}{\triangleright} \$ a$ hold for $Q_{2 \sim 4}$ in Figure 2. They all specify their corresponding $\$ t$ and $\$ a$ based on $\$ b$.

The variable binding dependency relationship satisfies:

$$
v_{i} \stackrel{p_{j}}{\triangleright} v_{j}, \quad v_{j} \stackrel{p_{k}}{\triangleright} v_{k} \Rightarrow v_{i} \stackrel{p_{j k}}{\triangleright *} v_{k} \text { (transitivity rule), }
$$

where $p_{j k}$ is the path expression obtained by concatenating $p_{j}$ and $p_{k}$, and $\triangleright *$ denotes an induced variable binding dependency. Given an XQuery, the direct variable binding dependencies extracted from it compose a base dependency set from which the non-direct dependencies can be derived inductively.

For example, $Q_{2}, Q_{3}$ and $Q_{4}$ in Figure 2 define $\$ b$ via an absolute path expression //book from the root of the source XML "bib.xml". Suppose variable $\$ r$ is used as a default root variable to be bound with the root element, then $\$ r \stackrel{\text { //book }}{\triangleright} \$ b$. Also since $\$ b \stackrel{\text { /title }}{\triangleright} \$ t$ and $\$ b \stackrel{\text { /author }}{\triangleright} \$ a$, we can derive $\$ r \stackrel{\text { //book/title }}{\triangleright *} \$ t$ and $\$ r \stackrel{\text { //book/author }}{\triangleright *} \$ a$. In contrast, $Q_{1}$ in Figure 2 directly defines $\$ t$ and $\$ a$ based on the root variable $\$ r$. Hence it has $\$ r \stackrel{/ / b o o k / t i t l e}{\triangleright} \$ t$ and $\$ r \stackrel{\text { //book/author }}{\triangleright} \$ a$.

\subsection{HMVD and XQuery Containment}

To tackle XQuery containment in the presence of variable binding dependencies, we cannot solely utilize tree homomorphism between the two respective navigation pattern trees. Additional conditions need to be asserted in the containment mapping process to deal with the effect of variable binding dependencies on the query semantics.

Let us consider the containment relationship between $Q_{1}$ and $Q_{2}$ in Figure 2 again. A tree embedding of the pattern tree of $Q_{1}$ into that of $Q_{2}$ exists, as illustrated by $E_{\text {body }}\left(Q_{1}, Q_{2}\right)$ in Figure 3. As described before, if we were to use $R_{Q 1}$ (see Figure 2) to answer $Q_{2}$ according to $Q_{2} \sqsubseteq Q_{1}$, then it will result in the superfluous answer pairs $t 1-a 1$, $t 1-a 2, t 3-a 1$ and $t 3-a 2$. With the new concepts introduced in this section, we can see that this is because the HMVD $\$ b \rightarrow \$ t \mid \$ a$ is required by $Q_{2}$ but not preserved by $Q_{1}$.

Suppose that $\operatorname{Vars}(Q)$ and $\operatorname{Rets}(Q)$ are the defined variables and the returned expressions in a query $Q$ respectively. All the variables occurring in $\operatorname{Rets}(Q)$ must be defined in $\operatorname{Vars}(Q)$ for $Q$ to be safe. On the other hand, variables occurring in $\operatorname{Rets}(Q)$ may be a subset of $\operatorname{Vars}(Q)$. That is, not all the variable binding dependencies are utilized in the query result. To determine query containment, we need to reason about not only the containment of the returned 
bindings due to $\operatorname{Rets}(Q)$, but also the containment of the utilized variable binding dependencies due to both $\operatorname{Var} s(Q)$ and $\operatorname{Rets}(Q)$. Correspondingly, we now define XQuery containment in the presence of variable binding dependencies.

Definition 2.3. Let $Q_{1}, Q_{2}$ be two XQueries. $Q_{1} \sqsubseteq$ $Q_{2}$ if and only if: 1) there exists a containment mapping from $\operatorname{Ret}\left(Q_{1}\right)$ to $\operatorname{Ret}\left(Q_{2}\right)$, and 2) the HMVDs preserved in $\operatorname{Vars}\left(Q_{1}\right)$ and utilized by $\operatorname{Ret}\left(Q_{1}\right)$ are subsumed by those preserved in $\operatorname{Vars}\left(Q_{2}\right)$ and utilized by $\operatorname{Ret}\left(Q_{2}\right)$.

For example, the HMVD $\$ b \rightarrow \$ t \mid \$ a$ is reflected in $R_{Q 2}$ in Figure 2 but not in $R_{Q 1}$. That is, the bindings of $\$ t_{Q 1}$ and $\$ a_{Q 1}$ are paired document-wise in $R_{Q 1}$, whereas those of $\$ t_{Q 2}$ and $\$ a_{Q 2}$ are grouped by their common book elements in $R_{Q 2}$. The former pairs can be derived from the latter by pairing all the $\$ t_{Q 1}$ bindings with $\$ a_{Q 1}$ bindings regardless of if they came from the same book parents. However, there is no way to recover the dependencies of $\$ t_{Q 1}$ and $\$ a_{Q 1}$ bindings on their common book elements as required by $Q_{2}$.

\section{OVERVIEW OF OUR APPROACH}

The main idea of our XQuery containment approach is to incorporate the checking of the containment of the utilized HMVDs in addition to the checking of the pattern tree homomorphism (i.e., the embedding of the containing query pattern tree into that of the contained query). The main steps of our approach are depicted in Figure 5.

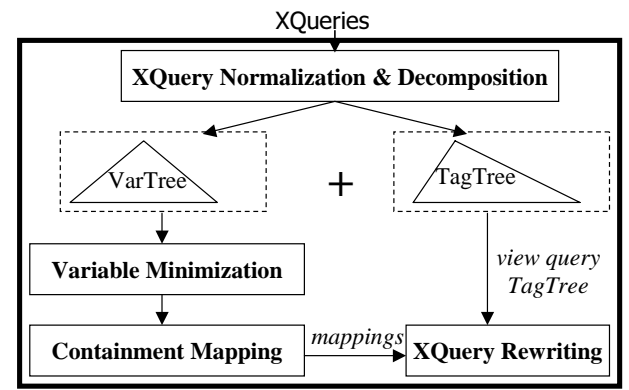

Figure 5: Containment Checking Flow

- XQuery decomposition. We separate the variable definition part from the result construction part and represent each using a tree structure. The former tree (i.e., VarTree) captures all the preserved HMVDs. It is different from the navigation pattern tree used in [23], as will be explained later. The latter tree (i.e., TagTree) is used to represent the result construction template. The TagTree also indicates how the preserved HMVDs are utilized in the result construction.

- Variable minimization. We identify the variables that are neither directly nor indirectly utilized in the result construction and degrade them to navigation steps. This way, we derive a minimal set of variable binding dependencies for which we conduct the containment checking. This is a critical step for ensuring the correctness of the containment result.

- Containment mapping. We conduct three types of containment mappings. First, we perform the minimal VarTree embedding to check the containment of the utilized HMVDs. Second, we check the tree embedding relationship between the navigation patterns. Lastly, we apply a mapping that deals with the effects of block-structure-induced variable dependencies on the containment of XQuery.

- XQuery rewriting. If the new query $Q_{1}$ is contained within a cached query $Q_{2}$, then the mapping $\mathcal{M}_{c}$ established in the containment mapping phase can be used for rewriting $Q_{1}$ against the query result structure of $Q_{2}$. The basic idea is to substitute each path expression $p$ in $Q_{1}$ for its corresponding path expression $p^{\prime}$ in the TagTree of $Q_{2}$ based on $p^{\prime}=\mathcal{M}_{c}(p) \circ \mathcal{M}_{t}$, where $\mathcal{M}_{t}$ represents the mapping of path expressions from the VarTree of $Q_{2}$ to its TagTree. Namely, $p^{\prime}$ is computed by the composition of $\mathcal{M}_{c}$ and $\mathcal{M}_{t}$. We skip the details of query rewriting in this paper.

\section{DECOMPOSITION AND MINIMIZATION}

\subsection{XQuery Decomposition}

The purpose of query decomposition is to separate the semantics of variable bindings from that of result construction. However, the semantic distinction is sometimes not very easily extracted from the surface syntax. For example, not necessarily all expressions in return clauses represent the return construction semantics. Due to the flexibility in composing a nested XQuery, FLWR expressions may be nested within a for clause, e.g., for $v_{2}$ in (for $v_{1}$ in $e_{1}$ return $e_{2}$ ) return $e_{3}$. In this case, $e_{2}$ in the nested return clause does not result in returning its bindings in the ultimate query result but only serves for specifying $v_{2}$ 's binding.

Therefore, we need to first normalize the query to derive a form based on which this semantic distinction is made easy. Then we represent the two semantics respectively using two tree structures, which are connected via variable bindings.

\subsubsection{The Normalization Rules in Use}

Our goal is that the normalized query can facilitate the separation of the path expressions that are to be output in the result from those that are used for specifying variable bindings, such that the later query decomposition step is made easy. There are a number of XQuery normalization techniques $[21,17,9]$ available. They overlap in some commonly used normalization rules. For example, unnesting the FLWR expression within a for clause (as illustrated before) is a standard rule shared by many techniques.

We adopt a set of query normalization rules including rules $(\mathrm{R} 2) \sim(\mathrm{R} 5),(\mathrm{R} 7) \sim(\mathrm{R} 10)$, and $(\mathrm{RG} 1)$ from [9]. We also apply rules (R1), (R6), (R11), and (R12), but in their reverse directions. Rule (R13) does not apply in our context since we exclude disjunctions from our XQuery fragment. Since we consider the XQuery fragment with no aggregations, we can also apply the rule that substitutes each let-variable with its definition. After applying these rules, the query is free of let clauses, empty sequence expressions and unit expressions. Also, only return clauses may contain nested FWR ${ }^{4}$ expressions.

\subsubsection{Decomposition into VarTree and TagTree}

DeFINITION 4.1. Given a normalized XQuery $Q$, a tree structure named VarTree $=(\boldsymbol{V}, \boldsymbol{E}, \boldsymbol{L})$ can be constructed based on the extracted variable binding dependencies. Each defined variable is denoted by a var node $v \in V$. Each

\footnotetext{
${ }^{4}$ Letter $L$ for representing let is removed since the normalized query is let-clause free.
} 
dependency $v_{i} \stackrel{p_{j}}{\triangleright} v_{j}$ corresponds to an edge $e=\left(v_{i}, v_{j}\right) \in E$ labeled $p_{j} \in L$. We refer to e the derivation edge of $v_{i}$.

The VarTree is different from the pattern tree concept referred to in other research [23]. An edge in the pattern tree corresponds to an axis step (/ or //) and the associated element type test. In contrast, a derivation edge in VarTree denotes the navigation pattern used for deriving a child variable from its parent. Actually this is indicated by the label on a derivation edge which is an XPath expression composed of possibly multiple steps and branches. In this sense, the VarTree can be considered as a nested tree with each edge encapsulating the navigation pattern corresponding to the label on it.

DeFInITION 4.2. For a normalized XQuery $Q$, a tree structure conforming to its nested block structure can be constructed to represent the result construction semantics. It is called TagTree $=(\boldsymbol{N}, \boldsymbol{A})$. Each block node $n \in N$ is a quadruple $[\bar{V}, \bar{C}, \bar{R}, \bar{T}]$ and each edge $a=\left(n_{i}, n_{j}\right) \in A$ denotes that block $n_{j}$ is nested within block $n_{i}$. Furthermore,

- $\bar{V}, \bar{C}, \bar{R}$, and $\bar{T}$ respectively represent the variables, where-conditions, return expressions, and to-be-constructed new elements specified in the corresponding block;

- $\bar{C}$ is denoted by a forest of constraint pattern trees each rooted at a variable defined in the local or an ancestor block. Equality conditions are associated with the corresponding node $(s)$;

- If unnesting of the bindings of variables in $\bar{V}$ results in a non-empty set and conditions $\bar{C}$ are satisfied, then the construction of a new element denoted by $\bar{T}$ will be invoked for each tuple in that unnested binding set;

- $\bar{T}$ may have either none, one, or a sequence of tag names in the form $\langle t 1\rangle\langle t 2\rangle \ldots\langle t n\rangle$. This means that the returns of $\bar{R}$ will be enclosed by an empty tag, $\langle t 1\rangle$ and $\langle/ t 1\rangle$, or $\langle t 1\rangle\langle t 2\rangle \ldots\langle t n\rangle$ and $\langle/ t n\rangle \ldots\langle/ t 2\rangle\langle/ t 1\rangle$.

We now extend the VarTree structure with a few more features. Given the TagTree $T T_{Q}$ of a query $Q$, we get each return expression $v / p_{m}$ in a $\bar{R}$ of $T T_{Q}$ and correspondingly attach to the var node for $v$ in the VarTree $V T_{Q}$ a leaf node (also referred to as ret node). Each ret node represents the corresponding return expression. To distinguish var nodes from ret nodes, we use solid circles to denote the former and use hollow circles for the latter.

The second extension is to shift the constraints and conditions in the $\bar{C}$ 's of $T T_{Q}$ to be represented in $V T_{Q}$. Specifically, if the constraint pattern represented by the XPath expressions (with or without variables defined in their respective where clauses ${ }^{5}$ ) is derived from $v$, or the equalitybased conditions are affiliated to where-variables that are dependent of $v$, then we move them in the filter "[ ]" of the XPath expression $p$ which labels the derivation edge of $v$ in $V T_{Q}$. Intuitively, this can be done because these constraints and conditions are, in a sense, analogous to the relational selection operations. They hence can be pushed to be executed in the navigation pattern matching stage for deriving variable bindings.

\footnotetext{
${ }^{5}$ Where-variables refer to variables defined in where clauses, while for-variables are those defined in for clauses. Unless indicated otherwise, "variable" means a for-variable. A where-variable can be removed since its life scope is refrained within the local where clause.
}

For example, the extended VarTrees and TagTrees of example $Q_{1}$ and $Q_{2}$ are depicted in Figure 6 respectively. Note that the where-condition "some $\$ p$ in $\$ b /$ price satisfies $\$ p=30$ " in the $\bar{C}$ of the bottom block in $T T_{Q 2}$ is serialized into "price $=30$ " and then moved in "[ ]" as the filter expression for defining $\$ b$ in $V T_{Q 2}$.
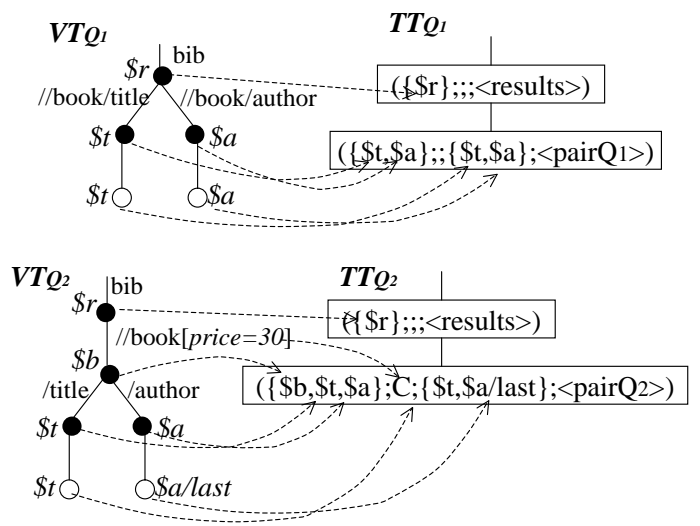

Figure 6: VarTrees and TagTrees of $Q_{1}$ and $Q_{2}$

However, we must carry out this VarTree extending process with caution. That is, the shifting of return expressions in $\bar{R}$ and where-conditions in $\bar{C}$ would not change the query semantics only if the to-be-attached var node $v$ is defined in the same block where $\bar{R}$ and $\bar{C}$ are specified. Some return expressions in $\bar{R}$ and where-conditions in $\bar{C}$ refer to variables that are defined in ancestor blocks. By moving them up along the nested block hierarchy to be attached to their referring variables, more or fewer bindings than desired may be returned. For example, suppose the example query $Q_{3}$ in Figure 1 also specifies the where-condition "some $\$ p$ in $\$ b /$ price satisfies $\$ p=30$ ", however in the inner block. Then attaching "[price=30]" to the definition expression of $\$ b$ in the outer block may cause generating fewer $<$ pair $Q 3>$ elements due to the push-up condition. We hence leave such return expressions and where-conditions in their original block nodes in $T T_{Q}$.

The VarTree with these extensions is comprehensive enough to also represent the to-be-returned bindings and the effect of where-conditions on variable bindings. It is also noteworthy that the VarTree and TagTree of a query are connected via variables. In particular, all variables in $\bar{V}$ 's and those referred to in $\bar{R}$ 's in the TagTree must be present as var nodes in the VarTree for the query to be safe.

\subsection{Use-based Variable Minimization}

We explained earlier that the VarTree of a query is a nested tree with navigation patterns encapsulated in its derivation edges. On one hand, the query semantics stays the same if we fully expand the VarTree by unnesting all the encapsulated navigation patterns and by naming each node in them with a variable. On the other hand, it is also possible not to affect the query semantics by degrading some variables into navigation pattern nodes to be encapsulated in derivation edges. We call the latter a variable minimization process since the number of var nodes is reduced (however with more complex navigation patterns encapsulated) and the VarTree structure seems minimized. A variable can be minimized without affecting the query semantics only if it does not participate in preserving any HMVD that is uti- 
lized in the result construction, nor serve in any way as a constraint context (will be explained later) for the return expressions.

Our goal here is to explore the opportunities for variable minimization to obtain the minimal VarTree (i.e., no further minimization is possible). This is critical since the later containment checking of utilized HMVDs can be based on the derived minimal VarTrees of two given queries.

DeFINITION 4.3. Given an XQuery $Q$, suppose $D$ is the source $X M L$ and $v$ is a variable defined in $Q$. If by substituting all occurrences of $v$ with $v$ 's definition, $Q$ 's result will not change for any XML data instance that conforms to the same DTD as D, then we say $v$ is nonessential. Otherwise $v$ is essential.

Now we provide practical criteria for distinguishing essential variables from non-essential ones based on their uses.

Explicit vs. Implicit Uses. A variable $v$ may either be used for defining another variable or in a return expression. We call the former case a Var use of $v$ and the latter a Ret use of $v$. Both are referred to as explicit uses of $v$ in general, regardless of where it is used (i.e., either in the local block where $v$ is defined or in descendant blocks).

Besides explicit uses, $v$ may also be implicitly used as a "loop counter" for invoking returns. For example, when the block where $v$ is defined encloses return expressions referring to other variables than $v$, then the cardinality of $v$ 's bindings is used to determine the number of times that the returns are to be invoked. In the extreme case when the binding set is empty (i.e., cardinality is 0 ), no return will be invoked. In this sense, $v$ serves as the constraint context for the returns.

If a variable $v$ has neither explicit nor implicit uses, we call it has no-use. Such variables are definitely nonessential and can be minimized. Otherwise, the essentiality of $v$ depends on the combination of different uses and the number of variable use occurrences.

One vs. Multiple Uses. Basically, $v$ is essential if it has at least two explicit uses, being either Var or Ret uses, or a Ret use and an implicit use. The detailed case studies and rationale are depicted in Figure 7.

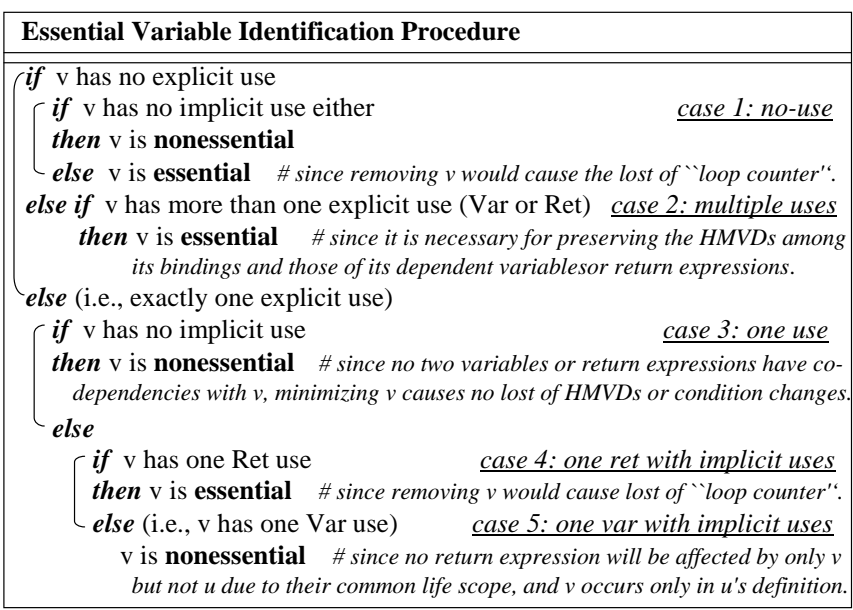

Figure 7: Identifying Variable Essentiality

Lemma 4.1. All essential variables can be correctly identified by our analysis in Figure 7 based on variable uses.
EXAmPle 4.1. We use $Q_{4}$ in Figure 2 to illustrate the minimization process. Before minimization (as shown in Figure 8), the var node for $\$ t_{Q 4}$ (denoted by the solid circles) in $V T_{Q 4}$ and that for $\$ a_{Q 4}$ each have one dependent ret node (denoted by the hollow circles). Hence $\$ t_{Q 4}$ and $\$ a_{Q 4}$ each have one Ret use. Also, the original TT $T_{Q 4}$ reveals that $\$ t_{Q 4}$ and $\$ a_{Q 4}$ each are specified alone in a bottom block. Thus they have no chance to affect any other return. This means that $\$ t_{Q 4}$ and $\$ a_{Q 4}$ each have no implicit use. Therefore, the var nodes for $\$ t_{Q 4}$ and $\$ a_{Q 4}$ in $V T_{Q 4}$ can be minimized according to the analysis of case 3 in Figure 7. Correspondingly, the XPath expressions on the ret nodes are changed to $\$ b /$ title and $\$ b /$ author respectively by substituting the variable occurrences by their definitions.

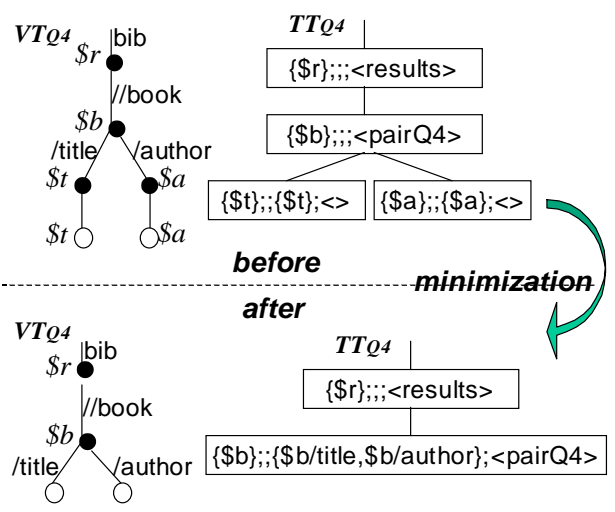

Figure 8: Minimization Example

\section{XQUERY CONTAINMENT MAPPING}

In this section, we present our containment mapping technique which is composed of three types of mappings. The first two mappings are based on the obtained minimal VarTrees, while the last one is based on the TagTrees.

\subsection{VarTree-based Containment Mapping}

Given two queries $Q_{1}$ and $Q_{2}$, the first mapping is to check the containment of the utilized HMVDs in two queries by conducting tree homomorphism (i.e., tree embedding) between their VarTrees. Suppose the embedding is from $V T_{Q 1}$ to $V T_{Q 2}$. Then the second mapping is to make sure that the navigation pattern used for deriving each var node in $V T_{Q 1}$ implies a more restricted constraint than that for the matched var node in $V T_{Q 2}$. These two mappings are called MAC mapping and MIC mapping respectively, indicating that the former is conducted at the macroscopic level of the VarTree (i.e., mapping of var nodes) while the latter is performed at the microscopic level of the VarTree (i.e., mapping of navigation patterns encapsulated in derivation edges).

\subsubsection{MAC Mapping}

We now extend the traditional tree homomorphism (namely based on root, label, and ancestor-descendant relationship preserving) to define the MAC mapping.

DeFinition 5.1. Suppose $V T_{1}$ and $V T_{2}$ are the minimal VarTrees of $Q_{1}$ and $Q_{2}$ respectively. For determining $Q_{1} \sqsubseteq$ $Q_{2}$, there must be a MAC mapping from $V T_{1}$ to $V T_{2}$, denoted by $\Phi\left(V T_{1}\right)=V T_{2}$, such that the following conditions are satisfied: 
$C 1) \operatorname{roots}\left(V T_{1}\right) \subseteq \operatorname{roots}\left(V T_{2}\right)$,

$C 2)$ for any node $u \in V T_{1}$, there is a match $\Phi(u) \in V T_{2}$ such that $\mathcal{T}(u)=\mathcal{T}(\Phi(u))$ if $\Phi(u)$ is a var node, and $\mathcal{T}(u)<: \mathcal{T}(\Phi(u))$ if $\Phi(u)$ is a ret node ( $\mathcal{T}$ returns the type of the element, and $<$ : denotes the subtypesupertype relationship),

C3) $u$ is an ancestor of $v$ for all $u, v \in V T_{1}$ if and only if $\Phi(u)$ is an ancestor of $\Phi(v)$ in $V T_{2}$, and

C4) if $u$ is a var node in $V T_{1}$, then $\Phi(u)$ is either a var or a ret node; if $u$ is a ret node, then $\Phi(u)$ must be a ret node.

Below we explain each of these required conditions.

C1: Root inclusion ${ }^{6}$. This condition requires that each source XML document referred to in $Q_{1}$ must also be referred to in $Q_{2}$. Correspondingly in the VarTrees, $\operatorname{roots}\left(V T_{1}\right)$ returns the URLs of the source XML documents involved in $Q_{1}$, which should be a subset of those returned by $\operatorname{roots}\left(V T_{2}\right)$.

C2: Mapping of element types. This condition requires a total but not necessarily injective mapping from nodes in $V T_{1}$ to those of $V T_{2}$. In addition, a node $u$ in $V T_{1}$ must be mapped to a node in $V T_{2}$ that has either the same type or a supertype ${ }^{7}$ of $u$ 's depending on if the matched node is a var node or a ret node. The element type of a node can be inferred from the XPath expression on its incoming derivation edge. $u$ can be mapped to a super-type ret node $\Phi(u)$ because the associated bindings of $\Phi(u)$ are all deeply returned (due to the semantics of a return expression) to enable the retrieval of $u$ 's bindings from subtrees of $\Phi(u)$ 's bindings in $Q_{2}$ 's result.

C3: Preservation of ancestorships. In a minimal VarTree, nodes represent essential variables and the HMVDs among them are captured by their ancestor-descendant relationships. Therefore, if all the ancestor-descendant relationships in $V T_{1}$ have correspondence mappings in $V T_{1}$, then it means that the to-be-utilized HMVDs required by $Q_{1}$ are all preserved by $Q_{2}$ and also present in $Q_{2}$ 's result.

C4: Correspondence of construct types. This condition checks the correspondence between query construct types. A var node represents a for expression while a ret node denotes a return expression. The bindings of a ret node are definitely returned whereas those of a var node may be used for constructing new elements correspondingly. Therefore, a var node can be mapped to a ret node and still get the correct bindings, while a ret node cannot be mapped to a var node since the new elements in $Q_{2}$ 's result rather than the original bindings would be returned in doing so.

We can see from the above conditions that the MAC mapping ensures that all the essential variable bindings, the HMVDs among them, and their attached returns required by $Q_{1}$ are preserved in the result of $Q_{2}$.

\subsubsection{MIC Mapping}

${ }^{6}$ Our technique allows a query to involve more than one XML document. In this case, the corresponding VarTree is actually a forest of trees, which may be connected by equality conditions on variables across trees.

${ }^{7}$ Here the concept of subtype-supertype is not the same as those in the object-oriented modeling domain. Instead, it corresponds to the element inclusion hierarchy in the DTD.
In addition to the MAC mapping, we need to check if the binding set of each node in $V T_{1}$ is indeed a subset of that of its match in $V T_{2}$. This is guaranteed by the MIC mapping, which essentially checks XPath containment.

Definition 5.2. Let $V T_{1}$ and $V T_{2}$ be the minimal VarTrees of $Q_{1}$ and $Q_{2}$ respectively. Suppose $\Phi\left(V T_{1}\right)=V T_{2}$ according to the MAC mapping. In MIC mapping, tree homomorphism is checked between the encapsulated navigation patterns for each pair of matched nodes. Two steps are carried out for each node $u$ in $V T_{1}$ :

1. If $u \notin \operatorname{roots}\left(V T_{1}\right)$, concatenate the XPath expressions along the path from $\Phi($ parent $(u))$ to $\Phi(u)$;

2. Assume that the XPath expression on the derivation edge of $u$ is $p_{1}$ and the one obtained from step (1) is $p_{2}$. $p_{1} \subseteq p_{2}$ is checked with $\subseteq$ denoting XPath containment (i.e., there is a tree homomorphism from the pattern tree representation of $p_{2}$ to that of $\left.p_{1}{ }^{8}\right)$.

Note that if a pair of parent-child nodes $(p, c)$ in $V T_{1}$ maps to a pair of ancestor-descendant nodes $(a, d)$ in $V T_{2}$ by the MAC mapping, then $p_{2}$ is the concatenated XPath expressions originated from $a$ to $d$. This implies that, to make $Q_{1} \sqsubseteq Q_{2}$ hold, more essential variables may be specified in $Q_{2}$ than $Q_{1}$ to preserve more HMVDs in $Q_{2}$ 's result. The MIC mapping makes sure that $p_{2}$, the XPath expression used for deriving $d$ 's bindings from $a$ 's, imposes a less restricted pattern constraint than $p_{1}$, the XPath expression used for deriving $c$ 's bindings from $p$ 's.

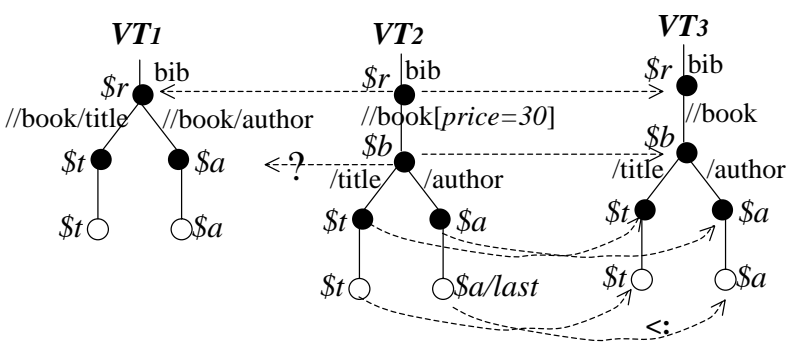

Figure 9: MAC Mapping between Minimal VarTrees

ExAmPle 5.1. Figure 9 illustrates two MAC mappings. One is between the two VarTrees of $Q_{1}$ and $Q_{2}$ in Figure 1. $\Phi\left(V T_{2}\right) \neq V T_{1}$ as shown on the left hand side. For one reason, the var node $\$ b$ in $V T_{2}$ has no match in $V T_{1}$ that satisfies $C 2$. We can hence derive $Q_{2} \nsubseteq Q_{1}$.

The second mapping is between the two VarTrees of $Q_{2}$ and $Q_{3}$ in Figure 1. The right hand side of Figure 1 shows $\Phi\left(V T_{2}\right)=V T_{3}$, i.e., $\Phi\left(\$ r_{Q 2}\right)=\$ r_{Q 3}, \Phi\left(\$ b_{Q 2}\right)=\$ b_{Q 3}, \Phi\left(\$ t_{Q 2}\right)=$ $\$ t_{Q 3}, \Phi\left(\$ a_{Q 2}\right)=\$ a_{Q 3}, \Phi\left(\$ t_{Q 2}^{\prime}\right)=\$ t_{Q 3}^{\prime}$, and $\Phi(\$ a /$ last $)=\$ a_{Q 3}^{\prime}$ $\left(\$ t_{Q 2}^{\prime}, \$ t_{Q 3}^{\prime}\right.$, and $\$ a_{Q 3}^{\prime}$ are the ret nodes). The mapping $\Phi(\$ a /$ last $)=\$ a_{Q 3}^{\prime}$ holds due to $\mathcal{T}(\$ a /$ last $)=$ last, $\mathcal{T}\left(\$ a_{Q 3}\right)=$ author, and last $\leq$ : author.

The MIC mapping between the navigation pattern trees encapsulated in the derivation edges of $V T_{2}$ and those of $V T_{3}$ is also successful. For example, the pattern tree for the $X P a t h$ expression "//book" in deriving $\$ b_{Q 3}$ can be embedded

\footnotetext{
${ }^{8}$ Our XQuery fragment allows XPath $\left(/ /{ }^{*},[]\right)$, for which the complexity of containment is CoNP-complete. However, the XPath containment complexity is reduced to PTIME if only two out of the three features are included. We refer the readers to [13] for the details of XPath containment.
} 
into that for "//book[price=30]" in deriving $\$ b_{Q 2}$. Note that the tree embedding direction for XPath containment $p_{1} \subseteq p_{2}$ is from $p_{2}$ to $p_{1}$.

\subsection{TagTree-based Containment Mapping}

We now address the implications of nested block structure on the containment of XQuery.

One intuitive example of such implications is the reliance of the return semantics on the emptiness of variable binding set(s). For example, note that since $Q_{2}$ in Figure 1 specifies both $\$ t_{Q 2}$ and $\$ a_{Q 2}$ in the outer block, the construction of a new $<$ pair $Q 2>$ element occurs only when a book element has both title and author subelements. In other words, if the binding set of $\$ a_{Q 2}$ is empty for a specific $\$ b_{Q 2}$ binding as for example for $b 1$ and $b 3$ in the source XML in Figure 2, then there will be no invocation of the return to construct the new elements.

Contrary to this example, the construction of $\langle$ pairQ3> elements for $Q_{3}$ in Figure 2 is solely based on the bindings of $\$ b_{Q 3}$, irrelevant of the bindings $\$ t_{Q 3}$ and $\$ a_{Q 3}$. The reason lies in the nested block structure of $Q_{3}$ (i.e., $Q_{3}$ has two query blocks versus that $Q_{2}$ has just one). While variables specified in $Q_{3}$ are the same as those in $Q_{2}$, they however are placed in different blocks (i.e., $\$ t_{Q 3}$ and $\$ a_{Q 3}$ are specified and returned in the inner block while $\$ b_{Q 3}$ is defined in the outer one) as oppose to being put in the same block as those in $Q_{2}$. Consequently, $Q_{2} \sqsubseteq Q_{3}$. Similarly, we have $Q_{3} \sqsubseteq Q_{4}$.

Recall that the TagTree structure of a query conforms to the nested block (see its definition in Definition 4.2). The $\bar{V}$ and $\bar{C}$ in an outer block together compose the evaluation context for those in its descendant blocks. Also, variables in the same $\bar{V}$ affect each other in the sense that their cartesian product would produce no tuple if any variable member in $\bar{V}$ has an empty binding set.

DeFinition 5.3. Let TT be the TagTree of query $Q$ and $n=[\bar{V}, \bar{C}, \bar{R}, \bar{T}]$ be a block node $n$ in it. Variables in $\bar{V}$ mutually depend on each other. Also, they all depend on those variables defined in n's ancestor block nodes. We call such dependencies region dependencies and denote them by $\hookrightarrow$.

Intuitively, if there is a variable binding $u \triangleright v$, then $v$ can only be defined either in the same block or a descendant block of where $u$ is defined, i.e., $u \hookrightarrow v$. However, we cannot imply $u \triangleright v$ from $u \hookrightarrow v$. This is formally stated as below.

Lemma 5.1. For any two variables $u$ and $v$ of $Q$, if $u \triangleright v$, then $u \hookrightarrow v$. $\square$

\subsubsection{Block Mapping}

We now define a third mapping that complements the previously defined MAC and MIC mappings.

DeFINITION 5.4. Let $T T_{1}$ and $T T_{2}$ be the TagTrees of $Q_{1}$ and $Q_{2}$ respectively. The block mapping is a one-to-many mapping function $\theta$ from each block node $n$ of $T T_{1}$ to nodes of $T T_{2}$, denoted by $\theta\left(T T_{1}\right)=T T_{2}$, such that $n=(\bar{V}, \bar{C}, \bar{R}, \bar{T})$ in $T T_{1}$ and its image set $S=\theta(n)$ in $T T_{2}$ satisfy:

$\left.C 1^{\prime}\right)$ for every variable $u \in \bar{V}, \Phi(u) \in \bigcup_{n_{i}} \bar{V}_{i}\left(n_{i} \in S\right)$,

$\left.C 2^{\prime}\right)$ for any two variables $w, x \in \bigcup_{n_{i}} \bar{V}_{i}\left(n_{i} \in S\right)$, if $w \hookrightarrow$ $x$, then there must be $u$ and $v$ in $T T_{1}$, such that $\Phi(u)=w, \Phi(v)=x, u \hookrightarrow v$, and

$\left.C 3^{\prime}\right)$ any $c_{i} \in \bigcup_{n_{i}} \bar{C}_{i}\left(n_{i} \in S\right)$ can be implied by a condition $c \in\left(\bar{C} \cup \bigcup_{m_{i}} \bar{C}^{\prime}\right) \quad\left(m_{i}\right.$ is an ancestor block node of $n$ ).
C1': Containment of variables. This condition is actually used for establishing the $\theta$ mappings (i.e., finding for each block node $n$ in $T T_{1}$ its image node set $S$ ) based on the VarTree node matches. Intuitively, a block node $n_{i}$ in $T T_{2}$ is included in $S$ if any variable in it is the match of any variable $u \in \bar{V}$ in $n . \bar{V}$ and $\bigcup_{n_{i}} \bar{V}_{i}$ denote variables in $n$ and the union of those defined in $n$ 's images $n_{i} \in S$ respectively.

C2': Implication of region dependencies. If a variable $w$ in an image node $n_{i}$ of $T T_{2}$ is involved in a region dependency (e.g., $w \hookrightarrow x$ ), then $\mathrm{C} 2$ ' ensures that there must be a region dependency between the corresponding variables in block nodes of $T T_{1}$. In other words, the region dependencies with matched variables in $T T_{2}$ involved must be a subset of those among the corresponding variables in $T T_{1}$.

C3': Implication of where-conditions. Suppose $n_{i}$ in $T T_{2}$ is an image node of the node block $n$ in $T T_{1}$. C3' checks if every where-condition $c_{i}$ left in $n_{i}$ can be implied from a where-condition $c$ either in $n$ or an ancestor block of $n$ (i.e., $c \in \bigcup_{m_{i}} \bar{C}^{\prime}$, with $m_{i} \in$ ancestors $(n)$ ).

In a nutshell, $C 1^{\prime} \sim C 3^{\prime}$ required by the block mapping make sure that $Q_{1}$ must assert more restricted constraints, i.e., region dependencies and where-conditions, than $Q_{2}$ does.

EXAMPle 5.2. Suppose that two adjacently nested blocks $n_{1}$ and $n_{2}$ in $T T_{1}$ define variables $u$ and $v$ respectively. There is no other variable in $n_{1}$ or in $n_{2}$. We also suppose that $\Phi(u)=x$ and $\Phi(v)=w$, and that $x$ and $w$ are defined in the same block $n$ in $T T_{2}$. By condition $C 1^{\prime}$, we have $\theta\left(n_{1}\right)=\{n\}$ and $\theta\left(n_{2}\right)=\{n\}$. We derive $x \hookrightarrow w$ and $w \hookrightarrow x$ due to the mutual region dependencies asserted by a block node. Also, from the enclosing relationship between $n_{1}$ and $n_{2}$, we know that $u \hookrightarrow v$ but $v \hookrightarrow u$. C2' is not satisfied based on these facts. Consequently, the block mapping fails and we derive $Q_{1} \nsubseteq Q_{2}$.

Putting all three types of containment mappings together, we now have a sound (not generally complete) solution for XQuery containment in the presence of variable binding dependencies.

Theorem 1. Given two XQueries $Q_{1}$ and $Q_{2}, Q_{1} \sqsubseteq Q_{2}$ if there exist a MAC mapping $\Phi\left(V T_{1}\right)=V T_{2}$, a MIC mapping $p 1 \subseteq p 2$ (i.e., the encapsulated XPath containment) for every matched node pair, and a block mapping $\theta\left(T T_{1}\right)=T T_{2}$.

\section{SYSTEM AND EVALUATION}

Based on our proposed containment mapping approach for XQuery, we have designed and implemented a semantic caching system called ACE-XQ [6, 7]. The ACE-XQ system is developed using Java 1.3. It utilizes the IPSI-XQ engine [14] installed at both the cache and the remote server sites to execute the rewritten query and the original query respectively. Source XML documents are hosted at the server.

The first set of experiments is for validating our containment mapping and rewriting methods for XQuery. For this, we designed some query workload ${ }^{9}$ that includes queries that are similar to those W3C use cases [20] and are within the scope of our XQuery fragment. The experiment shows that the results produced by running a query with and without

\footnotetext{
${ }^{9}$ We mainly focus on the "refining" case. Namely, the hit ratio of a new query being contained in a cached one is high.
} 


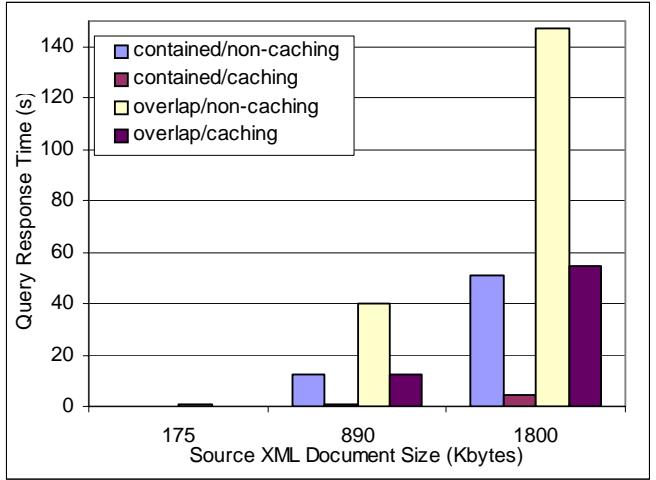

Figure 10: Query Response Times for Different Document Sizes w/o Caching

the attempt of conducting containment mapping and rewriting it against a containing view result are the same.

The second set of experiments is to evaluate the query performance with and without the semantic cache. As expected, Figure 10 shows the improved query performance by up to 10 folds for the totally contained cases in our setting.

Table 1 shows the break down of the query response time for a contained case into the computation overhead (i.e., query decomposition and minimization, containment mapping, and rewriting) and the query evaluation time. We see that the overhead is considerably small compared to the query evaluation time. This implies that although the complexity of our XQuery containment approach is NP-complete in general (since all three mappings are tree homomorphism extensions with additional checking of equivalence conditions, of the inclusive relationships between element types, etc.), it is efficient and practical in many real scenarios.

\begin{tabular}{|r|r|r|r|r|}
\hline $\begin{array}{r}\text { XML } \\
\text { Size }\end{array}$ & $\begin{array}{r}\text { Decomp. \& } \\
\text { Minimization }\end{array}$ & $\begin{array}{r}\text { Cont. } \\
\text { Mapping }\end{array}$ & $\begin{array}{r}\text { Query } \\
\text { Rewriting }\end{array}$ & $\begin{array}{r}\text { Query } \\
\text { Execution }\end{array}$ \\
\hline \hline $175 \mathrm{~KB}$ & $0.8 \mathrm{~ms}$ & $8.8 \mathrm{~ms}$ & $5.2 \mathrm{~ms}$ & $173.6 \mathrm{~ms}$ \\
$890 \mathrm{~KB}$ & $0.8 \mathrm{~ms}$ & $9.2 \mathrm{~ms}$ & $5.4 \mathrm{~ms}$ & $1068.8 \mathrm{~ms}$ \\
$1800 \mathrm{~KB}$ & $0.8 \mathrm{~ms}$ & $9.1 \mathrm{~ms}$ & $5.2 \mathrm{~ms}$ & $4525.4 \mathrm{~ms}$ \\
\hline
\end{tabular}

Table 1: Processing Time Decomposition

Extensive experimental studies can be found in $[8,5]$.

\section{CONCLUSION}

In this paper, we proposed a containment mapping approach that handles the effects of variable binding dependencies and the nested block structure on XQuery containment. Our approach provides sufficient conditions for solving nested XQuery containment.

An intermediate future work would be to incorporate the XQuery logical optimization technique in [9] in our normalization step to reduce the possible navigation redundancies in the VarTree representation. This helps to prune the space for conducting containment mapping. However, the lack of this optimization step as of now does not impact the soundness of the approach.

The XQuery fragment defined in this paper provides a good scope for us to focus on a set of important XQuery features with respect to the containment problem. We plan to extend the proposed containment mapping approach to accommodate a broader fragment of XQuery that includes disjunctions, aggregations, and other features as well as to consider more general constraints in XML and XQuery.

\section{REFERENCES}

[1] A. Deutsch and V. Tannen. Containment of Regular Path Expressions under Integrity Constraints. In KRDB, Rome, Italy, pages 1-11, June 2001.

[2] M. Arenas and L. Libkin. An Information-Theoretic Approach to Normal Forms for Relational and XML Data. In PODS, San Diego, CA, pages 15-26, 2003.

[3] D. Calvanese, D. Giacomo, and M. Lenzerini. Rewriting of Regular Expressions and Regular Path Queries. In PODS, Philadephia, PA, pages 194-204, 1999.

[4] A. K. Chandra and P. M. Merlin. Optimal Implementations of Conjunctive Queries in Relational Data Bases. In STOC, pages 77-90, 1977.

[5] L. Chen. A Semantic Caching System for XML Queries. Dissertation, WPI, 2003.

[6] L. Chen and E. A. Rundensteiner. ACE-XQ: A CachE-aware XQuery Answering System. In $W e b D B$, pages 31-36, June 2002.

[7] L. Chen, E. A. Rundensteiner, and S. Wang. XCache - A Semantic Caching System for XML Queries. In SIGMOD demonstration paper, Madison, Wisconsin, page 618, 2002.

[8] L. Chen, S. Wang, and E. A. Rundensteiner. Evaluation of Replacement Strategies for XML Query Cache. Data and Knowledge Engineering Journal (DKE), 49(2):145-175, 2004.

[9] A. Deutsch, Y. Papakonstantinou, and Y. Xu. The NEXT Logical Framework for XQuery. In $V L D B$, Toronto, Canada, pages 168-179, 2004.

[10] X. Dong, A. Halevy, and I. Tatarinov. Containment of nested xml queries. In $V L D B$, Toronto, Canada, pages 132-143, 2004

[11] W. Fan and J. Simeon. Integrity Constraints for XML. In PODS, Dallas, TX, pages 23-34, 2000.

[12] F.Neven and T.Schwentick. XPath Containment in The Presence of Disjunction, DTDs, and Variables. In ICDT, Siena, Italy, pages 315-329, 2003.

[13] G. Miklau and D. Suciu. Containment and Equivalence for an XPath Fragment. In PODS, Madison, Wisconsin, pages 65-76, June 2002.

[14] IPSI-XQ. http://ipsi.fhg.de/oasys/projects/ipsi-xq/index_e.html.

[15] D. Lee and W. W. Chu. Constraints-Preserving Transformation from XML Document Type Definition to Relational Schema. In ER, Salt Lake City, Utah, pages 323-338, 2000.

[16] A. Levy. Answering Queries Using Views: A Survey. VLDB Journal, pages 270-294, 2001.

[17] I. Manolescu, D. Florescu, and D. Kossmann. Answering XML Queries on Heterogeneous Data Sources. In $V L D B$, Roma, Italy, pages 241-250, 2001.

[18] I. Tatarinov and A. Halevy. Efficient Query Reformulation in Peer Data Management Systems. In SIGMOD, Paris, France, pages 539-550, 2004.

[19] M. Vincent and J. Liu. Multivalued Dependencies in XML. In BNCOD, Coventry, UK, pages 4-18, 2003.

[20] W3C. XML Query Use Cases, W3C Working Draft 02, May, 2003. http://www.w3.org/TR/xquery-use-cases.

[21] W3C. XQuery 1.0 and XPath 2.0 Formal Semantics. http://www.w3.org/TR/query-semantics/, May 2003.

[22] P. Wood. Containment for XPath Fragments under DTD Constraints. In ICDT, Siena, Italy, pages 300-314, 2003.

[23] S. A. Yahia, S. Cho, L. S. Lakshmanan, and D. Srivastava. Minimization of Tree Pattern Queries. In SIGMOD, Santa Barbara, CA, pages 315-331, 2001. 